\title{
Correspondence
}

Contents: Assessment of mental capacity: a dilemma/ Paracetamol overdose/Drug-induced dysphoria/Low incidence of mania in northern Finland/Seasonality of birth in Western Australia/Presenile dementia in a Down's syndrome adult with an unbalanced 21/21 Robertsonian translocation/Train of thought.

\section{Assessment of mental capacity: a dilemma}

SIR: At their joint conference in January 1996 the BMA and the Law Society launched the book Assessment of Mental Capacity - Guidance for Doctors and Lawyers. The ethical, legal and medical issues to be considered when competence to give a valid consent remains questionable constitute a great dilemma which is often faced by doctors in all specialities and especially by psychiatrists. Such a dilemma was encountered in the following case.

A surgeon diagnosed breast carcinoma in $\mathrm{D}$, a 48-year-old woman. He felt she was not competent to give consent for surgery as "she appears to be with the fairies". She showed evidence of psychosis but would not accept a psychiatric diagnosis or treatment and was therefore admitted under Section 2 (MHA 1983).

On admission there was evidence of pressure of speech, neologisms, formal thought disorder, thought broadcasting and a complex delusional system. At times she made no sense at all. Her mood was subjectively "fine" and objectively annoyed; her perceptions normal. Cognitively, she was fully orientated but refused further tests. She never mentioned the word "cancer", but knew the doctors called the "cyst" cancer. She refused treatment as she had no pain.

Past notes uncovered a diagnosis of schizophrenia 13 years earlier, when she was treated with neuroleptics but was lost to follow-up. Since then, from being a "normal person", she had deteriorated gradually.

Once cerebral pathology was excluded, she was prescribed Trifluoperazine. Six weeks after admission she asked "Have they confronted you about my bust? I'd like to discuss it with them." At this time, Section 3 MHA was instituted and although psychometric testing showed that she was unable to comprehend complex concepts, the surgeon and a second opinion felt that $\mathrm{D}$ was capable of giving consent to a total mastectomy.

She was discharged six weeks post-operatively, with diagnoses of schizophrenia and grade III infiltrating ductal carcinoma, and died within the next six months. D had been immediately sectioned to provide psychiatric care. Was six weeks too long to wait for potentially life-saving surgery? I hope that the new guidelines will help doctors with such dilemmas.

BMA and the Law Society (1995) Assessment of Mental Capacity Guidance for Doctors and Lawyers. Report of the British Medical Association and the Law Society. London: BMA Publications.

\section{Shrewsbury Child and Family Service \\ Radbrook Centre}

M. PAUL

Shrewsbury SY3 9BL

\section{Paracetamol overdose}

SIR: I wish to comment on the paper by Hawton et al (1996) concerning paracetamol self-poisoning. We have carried out a similar study in Leicester on 174 people following paracetamol overdose. Our findings are broadly in agreement with the Oxford study. Forty-one per cent of patients correctly identified liver failure as the danger of paracetamol overdose. We investigated the use of warning labels and it appears these would only have a marginal effect on overdose behaviour. The most important finding was that of the 14 large overdoses of 50 tablets or more, 13 were from bottles and only one from blister packs. We will be reporting these findings in detail elsewhere but would wish to agree that the change in packaging from large bottles to blister packs would probably bring about a reduction in serious medical complications and the overall number of suicides in people using paracetamol.

\footnotetext{
hawton, K., Ware, C., Mistry, H., et al (1996) Paracetamol self-poisoning - characteristics, prevention and harm reduction. British Journal of Psychiatry, 168, 43-48.
}

T. FRIEDMAN

Leicestershire Mental Health Service NHS Trust

Gwendolen Road

Leicester LES 4PW 